\title{
Discapacidad y sexualidad en Europa. Hacia la construcción del acompañamiento sexual
}

\author{
Disability and sexuality in Europe. Towards the construction of sexual \\ accompaniment
}

\begin{abstract}
Resumen
El presente artículo plantea uno de los rodeos analítico-reflexivos devenidos de la investigación en proceso de elaboración 'Cuerpos vivos, cuerpos sentidos. Acompañamiento sexual para personas en situación de discapacidad con dependencia severa'. El objetivo de la misma radica en analizar la relación discapacidad-sexualidad, con la especificidad en la deconstrucción del rol y figura del/de la acompañante sexual. La metodología que se está implementando da cuenta de entrevistas a representantes de la sociedad civil organizada y de la arena política en torno a la temática en Europa (junio 2017-julio 2018) y en América Latina (mayo 2018-junio 2019). Se presenta aquí el trabajo de campo realizado en Europa y sus primeros análisis en torno a las luchas por el reconocimiento. Desde allí se orienta la discusión hacia los marcos normativos de estos países para comprender el papel del Estado en este entramado, en su conjunción con la sociedad civil organizada.
\end{abstract}

\section{Palabras clave}

Discapacidad, sexualidad, acompañamiento sexual, reconocimiento.

\begin{abstract}
The present article proposes one of the analytical-reflexive detours that have emerged from the research being developed 'Living bodies, sensed bodies. Sexual accompaniment for people in situation of disability with severe dependence'. The objective is to analyze the relationship between disability-sexuality, with the specificity in the deconstruction of the role and figure of the sexual companion. The methodology that is being implemented reports on interviews with representatives of organized civil society and the political arena around the thematic in Europe (June 2017-July 2018) and in Latin America (May 2018-June 2019). It presents the field work carried out in Europe and its first analyses around of recognition struggles. From there, the discussion is oriented towards the normative frameworks of these countries in order to understand the role of the State in this framework, in conjunction with organized civil society.
\end{abstract}

\section{Keywords}

Disability, sexuality, sexual accompaniment, recognition.

\author{
María Noel Míguez Passada \\ $<$ marianoel.miguez@ \\ cienciassociales.edu.uy>
}

Universidad de la República. Uruguay

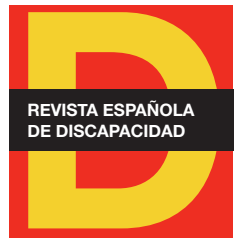

Para citar:

Míguez, M. N. (2019): “Discapacidad y sexualidad en Europa. Hacia la construcción del acompañamiento sexual". Revista Española de Discapacidad, 7 (I): 133-152.

Doi: <https://doi.org/10.5569/23405104.07.01.07>

Fecha de recepción: 05-10-2018 Fecha de aceptación: 02-04-2019 
"Todo cambia si se considera que la sociedad se presenta para cada cual, como una perspectiva de porvenir, y que este porvenir penetra en el corazón de cada cual como una motivación real de sus conductas"

(Sartre, 2000: 81).

\section{Introducción}

El presente artículo se enmarca en la investigación que se está llevando adelante como integrante de la Cohorte 2018 del Posdoctorado en Ciencias Sociales de la Universidad de Buenos Aires, Argentina. La misma versa sobre la relación discapacidad-sexualidad, con la especificidad del rol y figura del/de la acompañante sexual para personas en situación de discapacidad con dependencia severa en Europa y América Latina.

El objetivo general de dicha investigación es analizar la relación discapacidad-sexualidad en el reconocimiento de la sexualidad de las personas en situación de discapacidad con dependencia severa en Europa y América Latina, siendo sus objetivos específicos: deconstruir los marcos teóricos en torno a la relación discapacidad-sexualidad, conocer el estado del arte de la temática en Europa y América Latina, y generar insumos en torno a sensaciones y percepciones de la población objetivo de la presente investigación para una reflexión crítica del rol y figura del acompañamiento sexual para personas en situación de discapacidad con dependencia severa. De esta manera, se pretende no sólo conocer el estado del arte de la temática en los países del muestreo de ambos continentes, sino producir conocimiento en torno a la misma que permita hacerla visible, analizarla críticamente y generar sensibilización e información.

Para los fines del presente artículo, se reflexiona sobre parte de los datos surgidos del trabajo de campo llevado adelante en Europa. Ello implicó realizar recortes en varios rodeos analíticos ${ }^{1}$ y en la bastedad de datos recabados, los cuales están siendo materializados en otros trabajos.

En lo que hace a la temática de la discapacidad en su relacionamiento con la sexualidad, la misma ha comenzado a tener mayor visibilidad en los últimos años en el mundo occidental. Varias han sido las luchas por el reconocimiento ${ }^{2}$ de la sociedad civil organizada para poner en el tapete, no sólo los aspectos que hacen a la sexualidad como parte constitutiva de la condición humana, sino en torno a aspectos que se dirimen en la concreción de ello para personas en situación de discapacidad con dependencia severa ${ }^{3}$. Esto ha puesto luz a una temática históricamente invisibilizada, tomada como tabú, desconocida para la mayoría de las personas que poco o nada de contacto tienen con esta población. En este sentido, más allá de que la concreción de la sexualidad en personas en situación de discapacidad con dependencia severa se haya ido asumiendo

1. Por ejemplo, dejando fuera de este artículo delimitaciones en torno a miradas feministas, análisis desde la teoría crip, la cuestión LGTBIQ, entre otros.

2. “...se llega a ser un sujeto individual únicamente cuando se reconoce y se es reconocido por otro sujeto. El reconocimiento de los otros, por lo tanto, es esencial para el desarrollo del sentido de sí. No ser reconocido -o ser reconocido inadecuadamente- supone sufrir simultáneamente una distorsión en la relación que uno mantiene consigo mismo y un daño infligido en contra de la propia identidad" (Fraser, 2000: 57).

3. La dependencia severa se define como: "Ya sea que se trate de discapacidades físicas, intelectuales, mentales o múltiples, con la condición de que necesiten de otra persona en forma permanente, para poder desarrollar los actos corrientes de la vida diaria" (MIDES, 2014: 2) Para los fines presentes, se hace referencia a todo tipo de discapacidad con dependencia severa: física, mental, intelectual, sensorial. 
en sus diversas formas desde siempre, en Europa ha surgido en la última década el rol y figura del/de la acompañante sexual ${ }^{4}$. Esta nueva figura, con su rol específico, es parte constitutiva de la deconstrucción analítica que se propone en el presente artículo, de forma tal de poder ir desandando preguntas que han ido surgiendo, a saber: ¿qué implica el acompañamiento sexual?, ¿qué países lo están llevando adelante y en qué condiciones?, ¿desde qué perspectiva de sexualidad y discapacidad?, ¿aparece este rol y figura a partir de luchas por el reconocimiento de las personas en situación de discapacidad? Estas y otras tantas preguntas surgen a la hora de comenzar a imbuirse en la temática en un contexto donde varias son las miradas, las luchas y las concreciones que se han venido dando en la última década.

El posicionamiento teórico-metodológico está dado desde el modelo social de investigación y/o intervención de la discapacidad, en superación con las posturas rehabilitadoras e individuales del modelo médico. Ello se basa en el reconocimiento de la discapacidad como temática colectiva, y no como problemática individual, apuntando a los derechos y a la igualdad como determinaciones sustanciales para el despliegue singular y colectivo, no sólo de las personas en situación de discapacidad directamente interpeladas, sino por la sociedad en su conjunto.

La lógica de exposición que se llevará adelante en el presente artículo da cuenta de un primer punto en el cual se desarrolla el mapeamiento del campo de investigación en la especificidad europea, para dar lugar al estado del arte de la temática en dicho continente, en lo que se entienden dos aristas sustanciales: las luchas por el reconocimiento de la sociedad civil organizada para el logro de derechos, por un lado, y los marcos normativos que se materializan (o no) a partir de tales demandas, por el otro. Se cierra el artículo con reflexiones finales que apuntan a generar nuevas aperturas en la temática.

\section{Mapeamiento del campo}

La especificidad de los marcos teóricos de discapacidad (modelo social) y sexualidad (como inherente a la condición humana) se hacen sustanciales para poder desandar la temática desde un análisis crítico de la misma, anclado en la sexualidad como derecho para todos y todas, más allá de la situación singular de cada sujeto.

Cuando se hace referencia al modelo social de investigación y/o intervención en discapacidad ${ }^{5}$ se orienta la temática como problemática social y se pone el foco en la condición de 'persona' de aquellos sujetos ubicados en la alteridad a partir de una ideología de la normalidad ${ }^{6}$ que traza líneas demarcatorias entre lo

4. En las entrevistas realizadas, las formas de nombrar que aparecieron fueron: acompañamiento sexual, asistencia sexual, y acompañamiento íntimo y erótico. Para los fines presentes, se utiliza acompañamiento sexual, más allá se esté en un proceso de resignificación de esta forma de nombrar para hallar un correlato latinoamericano que sea identificado como propio.

5. “...la perspectiva del modelo social (...) permite trascender miradas unilineales y clásicas propias del modelo médico. En este sentido, la discapacidad es entendida como una construcción social basada en términos de desigualdad, donde las líneas demarcatorias entre normalidad y anormalidad responden a posturas etnocéntricas. Por ello urge reconocer el carácter social y no natural de la discapacidad, para superar perspectivas hegemónicas que se continúan produciendo y reproduciendo en estas sociedades (...). Por ello, la discapacidad como construcción social hunde sus raíces en la superación de la producción de la alteridad con base en la desigualdad" (Míguez et al., 2015: 15).

6. "La ideología de la normalidad halla su espacio en el marco de los procesos de producción y reproducción de líneas demarcatorias tendientes a generar relaciones de nosotros-otros, normales-anormales, en lo que sería la disputa por la temática de la discapacidad en su distinción del 
normal y lo anormal, y que atraviesa la vida cotidiana de cada uno de los sujetos en sociedad. Interiorizar la norma estandarizada como la hegemónicamente válida lleva a demarcar, opuesta y complementariamente, a aquellos que no la siguen según lo estipulado. La discapacidad, desde una perspectiva social, trasciende lo fenoménico de tal ideología y la deconstruye hacia el reconocimiento, la igualdad, los derechos y la escucha atenta de aquellos que quedan en la disidencia por su silencio (por acción, por omisión y/o por restricción).

Desde este posicionamiento en torno a la discapacidad se hace plausible la incorporación de la sexualidad en el entendido que esta es parte constitutiva del ser, por un lado, y que en la especificidad de esta relación urge superar más aún los componentes de la ideología de la normalidad que trazan diagnósticos con pronósticos, constriñen posibilidades y potencialidades, reduciendo a los sujetos concretos a su 'déficit' en lugar de ampliarlo hacia sus derechos. Por ello, es parte constitutiva de la vida de cada sujeto, siendo un aspecto natural y necesario a ser desarrollado, y un derecho fundamental a ser ejercido. Desde esta perspectiva, la relación sexualidad-discapacidad incluye la condición de sujeto de derecho de toda persona más allá de su situación singular, promoviendo la dignidad, la libertad, la intimidad, la integridad física, psicológica y moral, entre otras tantas cuestiones. Ello, en el entendido de que "la renuncia al ejercicio de la propia sexualidad sólo puede ser fruto de una decisión libre: nunca puede ser impuesta por otras personas" (Tandem-Team, 2016: 63).

En la especificidad de este artículo, en el trabajo de campo realizado en Europa, se destacan las entrevistas realizadas a informantes calificados de movimientos por el reconocimiento de la sexualidad para personas en situación de discapacidad, como son: Claudine Damarc (presidenta de Cuerpos Solidarios, Ginebra -Suiza-), Francesc Granja (presidente de Tandem-Team, Barcelona -España-), Antonio Centeno (codirector del film 'Yes, we fuck' y activista en la temática, Barcelona -España-), Rafael Reoyo (académico y activista, Barcelona -España-), Steven De Wirdt (presidente de Aditi -organización civil de lucha a favor de la sexualidad para personas con dependencia severa-, Bruselas -Bélgica-), Sheila Waremburg (presidenta de Sexual Understanding, Annecy -Francia-), Marcel Nuss (activista por la legalización de la asistencia sexual para personas en situación de discapacidad con dependencia severa en Francia, Montpellier -Francia-), Thomas Shakespeare (académico y activista en situación de discapacidad con dependencia severa, Norwich -Inglaterra-), 'Joke' (acompañante sexual, Passieflower, Amsterdam -Holanda-) e 'Isabelle' (acompañante sexual, Montpellier -Francia-). Sin ser Thomas Shakespeare, académico británico con quien se tiene vínculo por el programa 'Handicap et Société' de la Escuela de Altos Estudios en Ciencias Sociales, de París, al resto de los entrevistados se llegó a través de la página online de la European Platform Sexual Assistance $(\text { EPSEAS })^{7}$, portal para el acceso a diversas organizaciones europeas que trabajan la temática de la discapacidad y la sexualidad y, específicamente, el rol y figura del/de la acompañante sexual. Este portal fue creado por Steven De Wirdt, según se supo en la entrevista que se le realizó en Bruselas en junio de 2018, con el objetivo de contribuir al debate público y político y sensibilizar a la opinión pública sobre la relevancia de la sexualidad en esta población, así como para generar una plataforma que reúna las distintas miradas en los países europeos.

modelo social y el modelo médico. Reconocer que existe una ideología de la normalidad que revela la inscripción de demarcaciones societales entre sujetos concretos permite analizar la temática desde el modelo social de la discapacidad. Se entiende que solo si se reconoce su existencia se podrán superar sustancialmente las relaciones de desigualdad inmanentes a la situación de discapacidad de hoy" (Míguez et al., 2015: 16).

7. La EPSEAS se refiere a la relación sexualidad-discapacidad para personas en situación de discapacidad en general. Nótese que la población objetivo de esta investigación se reduce a las personas en situación de discapacidad con dependencia severa, población que abarca la EPSEAS, pero que supera en su extensión. 
De esta manera, los países seleccionados para el muestreo europeo dan cuenta de los surgidos en el marco de EPSEAS. Con ello no se desconoce la existencia de otros espacios dentro de los mismos países del muestreo, así como de otros países de Europa ${ }^{8}$.

La Unión Europea ratificó en enero de 2011 la Convención sobre los Derechos de las Personas con Discapacidad (CDPD) de Naciones Unidas, del año 2006, que parte del principio de que las personas en situación de discapacidad son sujetos de derechos y obligaciones en igualdad de condiciones que el resto de la sociedad. En la especificidad de la sexualidad no se trata del reconocimiento del derecho a la sexualidad, el cual se da por descontado, sino de su encuadre y dispositivos necesarios y específicos para su materialización.

Para las personas en situación de discapacidad con dependencia severa, la ausencia de marcos normativos apropiados ha sido la razón sustancial para la falta de reconocimiento y respeto del ejercicio pleno de sus derechos sexuales, siendo atravesadas sus vidas cotidianas, por lo general, por la complejidad en el manejo (propio y de terceros) de sus cuerpos y el contacto con personas que no sean de su entramado familiar, social y/o profesional. En este contexto surge la figura del acompañamiento sexual para personas en situación de discapacidad mayores de edad, en el reconocimiento de que cada persona y su sexualidad son únicas. Por ende, cada encuentro consensuado es el resultado de circunstancias únicas, donde el entramado sexualidad-discapacidad se acompasa con el desafío de conocer sensaciones, percepciones, potencialidades corporales, placer, autonomía, etc.

\section{Luchas por el reconocimiento}

El concepto de luchas por el reconocimiento se toma desde la teoría del reconocimiento, puesta en danza por Axel Honneth hace un cuarto de siglo a partir de recuperar las ideas centrales de Hegel en sus primeros años de juventud (período de Jena). Los puntos medulares que Honneth (1997) retoma de Hegel dan cuenta de tres esferas del reconocimiento, las cuales son necesarias para generar relaciones intersubjetivas ${ }^{9}$ de $^{2}$ los sujetos en sociedad basadas en el respeto e igualdad, a saber: amor, derecho y solidaridad. La primera $\left(a m o r^{10}\right)$ resulta de un equilibrio precario entre autonomía y conexión en los espacios más íntimos del relacionamiento humano, la segunda (derecho ${ }^{11}$ ) apunta al sentirse parte de un todo generalizado como lo es el

8. A través de una de las entrevistas realizadas en Barcelona en junio de 2018 surge la información que en Italia también existe una organización que lucha por la figura del acompañamiento sexual para personas en situación de discapacidad. Similar fue la situación con la organización Plena Inclusión, de España. De todas formas, en virtud de que el trabajo de campo estaba para finalizar en Europa (período junio 2017-julio 2018) se tomó la decisión metodológica de no continuar ampliando la información, quedando para otra investigación los datos a recuperar de dicho país. Por lo pronto, se dejan las páginas web en caso de interés: https://www.lovegiver.it/, http://www.plenainclusion.org/.

9. Más allá Honneth (1997) no se refiere específicamente a intersubjetividad, desde la presente investigación se plantea este concepto que se entiende sustancial. Para ello, se recupera a Sartre en sus ideas, a saber: "El otro es indispensable a mi existencia tanto como el conocimiento que tengo de mí mismo (...). Así descubrimos en seguida un mundo que llamaremos la intersubjetividad, y en este mundo el hombre decide lo que es y lo que son los otros" (1947: 40).

10. “...en su culminación los sujetos recíprocamente se confirman en su naturaleza necesitada y se reconocen como entes de necesidad; en la experiencia recíproca de atención amorosa los dos sujetos se saben unificados, porque en su necesidad son dependientes del otro ocasional (...). Con ello se dice que las relaciones afectivas primarias están destinadas a un equilibrio precario entre autonomía y conexión" (Honneth, 1997: 118). 11. "Sólo desde la perspectiva normativa de un "otro generalizado" podemos entendernos a nosotros mismos como personas de derecho, (...) (esto) permite comprender la relación de derecho como una forma de reconocimiento recíproco" (Honneth, 1997: 132). 
derecho positivo, la tercera (solidaridad ${ }^{12}$ ) da cuenta de cómo la sociedad interpela las singularidades. De esta manera, se puede estar reconocido en una $u$ otra de las esferas, siendo el reconocimiento en las tres el punto fundamental para generar autonomía de los sujetos en sociedad. El falso reconocimiento o el menosprecio, así nombrados por este autor, aparecen cuando alguna de estas esferas se ve interpelada por una intersubjetividad que reproduce relaciones de desigualdad y vulnera los procesos de autonomía singulares y/o colectivos. En las situaciones de discapacidad, el falso reconocimiento o el menosprecio terminan siendo moneda corriente en sus cursos de vida.

La relación sexualidad-discapacidad se ve transversalizada por las tres esferas del reconocimiento. En cuanto a la esfera del amor, las relaciones íntimas e intersubjetivas, en su generalidad, se despliegan en la vida cotidiana de las personas en situación de discapacidad en un quiebre del "equilibrio precario" entre autonomía y conexión, quedando en una eterna dependencia física y emocional de aquellos autopercibidos como 'normales'. Ello se potencia con mayor intensidad en las situaciones de dependencia severa. Esta esfera es sustancial en los primeros años de sociabilidad para generar procesos de autonomía real en los sujetos. La relación sexualidad-discapacidad comienza a encontrar los primeros obstáculos y conformación de falsos reconocimientos o menosprecios en estos primeros años de sociabilidad, que marcarán fuertemente el curso de vida de las personas en situación de discapacidad. El "no puede" como palabra corriente se diluye y expande en la vida cotidiana de estos sujetos, quienes se piensan desde el "yo normalizado" como niños/ as eternos y asexuados. Con ello, se pierde de vista que la sexualidad es parte de la vida cotidiana y que se va desplegando en la misma al igual que otros aspectos de la sociabilidad. Se pone el foco en lo coital, se desplaza el 'problema' hacia las edades adultas de esta población (en el mejor de los casos, sino ni siquiera se los piensa como seres sexuados) y se convierte en un problema a resolver, fundamentalmente, por el ámbito médico. Si en la esfera del amor el reconocimiento de la relación sexualidad-discapacidad transita como parte del proceso de sociabilidad, la potencia de generar autonomía de las personas en situación de discapacidad amplía el campo de sus posibles ${ }^{13}$ hacia concreciones corporales, sensoriales y emocionales de respeto por sus propias corporalidades y las de los otros.

Con relación a la esfera del derecho en la relación sexualidad-discapacidad, la generación de un marco normativo que contemple la sexualidad como parte constitutiva y sustancial de los sujetos en sociedad aportaría el reconocimiento necesario para generar autonomía en esta interrelación. La sexualidad entendida como uno de los aspectos fundamentales de todos los sujetos, y en la especificidad de las personas en situación de discapacidad con dependencia severa como dependientes no sólo de un otro que le habilite a desarrollar las actividades básicas de su vida cotidiana, sino un "otro generalizado" a través del derecho positivo que los reconozca como seres sexuales y sexuados, generando, por ejemplo, la figura del acompañamiento sexual. Con el despliegue del control de los cuerpos y las sensibilidades en la modernidad, la sexualidad quedó remitida a lo reproductivo (como especie) y, por ende, a lo netamente coital, bajo el requerimiento de la creación de sujetos eficaces y eficientes para la lógica del capital. Los deseos y pasiones fueron quedando mermados a los controles biopolíticos y anatomopolíticos (Foucault, 1998), donde cualquier gasto de energía que no fuese para el trabajo se fue considerando patológico. Si a esta mirada de la sexualidad, se le suma la de la discapacidad construida en el siglo XX, el resultado es absolutamente restrictivo para esta población: seres asexuados e infantilizados, que en caso de exacerbar sus pasiones se los reprime con psicofármacos

12. "Para poder conseguir una ininterrumpida autorrelación, los sujetos humanos necesitan, más allá de la experiencia de la dedicación afectiva y del reconocimiento jurídico, una valoración social que les permite referirse positivamente a sus cualidades y facultades concretas" (Honneth, 1997: 148). 13. "El campo de los posibles es así el fin hacia el cual supera el agente su situación objetiva. (...) Pero por muy reducido que sea, el campo de lo posible existe siempre" (Sartre, 2000: 79). 
para reducir los efectos libidinales y se coarta toda posibilidad de reproducción biológica (la más común es la esterilización femenina, en la mayoría de las veces sin consentimiento de la mujer en situación de discapacidad). Crear una figura en los marcos normativos podría ser una posibilidad de desandar este entramado restrictivo y reubicar la temática de la sexualidad en lo que es: el despliegue de los deseos, sensaciones y percepciones corporales y emocionales en la vida cotidiana de los sujetos.

En lo que hace a la esfera de la solidaridad en su correlato con la relación sexualidad-discapacidad, en principio implicaría deconstruir la idea genérica que se continúa reproduciendo de la sexualidad como lo netamente coital, en una búsqueda del placer eficaz y eficiente, de lo que se interioriza en sociedad como lo que sería vivir 'normalmente'. Si se entiende la sexualidad como parte del proceso de sociabilidad, donde la intersubjetividad es constitutiva de la interdependencia que genera vivir en sociedad, se llegaría a desplazar la idea netamente coital de la sexualidad y se podría comprender en el marco de la afectividad humana. En este aspecto no hay distinción entre seres etiquetados como 'normales' o 'anormales', puesto que la exteriorización de estas interiorizaciones (Sartre, 2000) dan cuenta de la generalidad humana de estos tiempos. Lo que sucede es que, en las situaciones de discapacidad, y más aún de dependencia severa, esto se potencia hasta un constreñimiento casi ilimitado de estos cuerpos y corporalidades. Las sociedades modernas tienen demasiado para deconstruir en torno a la sexualidad, y más aún en su relación con la discapacidad.

Tal como se ha planteado, cuando estas esferas del reconocimiento no se dan, aparece el falso reconocimiento o el menosprecio, generando más dolores y sinsabores en los sujetos que por estos aspectos quedan interpelados. Surge en este entramado lo que Honneth (1997) llama luchas por el reconocimiento. A partir de experiencias efectivas de dominación de menosprecio sufrido surge la posibilidad de la lucha, la cual es ocasionada afectivamente por las emociones negativas que acompañan la experiencia del desprecio. La lucha, tal como la retoma Honneth de Hegel, revela un acercamiento social, no expresando un conflicto universal sino práctico. Es el medio por el cual un sujeto busca ser reconocido por otro sujeto en sus aspectos específicos. De esta manera, la "lucha social" es un "proceso práctico a través del cual las experiencias individuales de desprecio son interpretadas como experiencias típicas de un grupo entero, de manera de motivar la reivindicación colectiva a través de relaciones de reconocimiento" (Honneth, 1997: 194). Comienzan así a generarse movimientos colectivos en torno a la superación de situaciones de falso reconocimiento o de menosprecio. En la relación sexualidad-discapacidad, surge en varios países europeos (así como en algunos estados de Estados Unidos y en Australia ${ }^{14}$ ) la reivindicación en la última década del rol y figura del acompañamiento sexual para personas en situación de discapacidad: "El movimiento de personas con discapacidad ha sido llamado "el último movimiento de liberación": se basa en, y adopta las tácticas del movimiento de derechos civiles, movimientos de mujeres, lesbianas y gays, que le precedieron" (Shakespeare, 2018: 144).

En Suiza, a comienzos del año 2000 empieza a reunirse el primer grupo de personas en situación de discapacidad para trabajar la temática de la asistencia sexual ${ }^{15}$, en el marco de la Asociación Sexualidad y discapacidad plural, realizándose ocho años después un primer curso de formación en torno a este rol y figura. A partir de esta asociación surgen otras en el país (fundamentalmente en los cantones francófonos), conformándose en 2009 una de las más importantes de hoy día: Cuerpos Solidarios. A través de esta se habilita un espacio de encuentro para toda persona que quiera participar en la construcción de este rol y figura, estando

14. Se suman EEUU y Australia, más no se tomó contacto con movimientos en dichos países, centrándose el trabajo de campo de esta investigación en la mayoría de los países europeos que han venido luchando por el reconocimiento de esta temática en la última década.

15. Ante el énfasis de los distintos movimientos de personas en situación de discapacidad en torno a la forma de nombrar de este rol y figura, se opta por nombrar según lo ha hecho cada espacio en su particularidad. 
su comité directivo conformado por al menos tres asistentes sexuales de los cinco que lo integran. En 2013 crean su sitio de internet y realizan la primera formación de asistentes sexuales. La distinción que remarca Cuerpos Solidarios de la Asociación Sexualidad y discapacidad plural se centra en que aquéllos apuntan a un seguimiento psicológico en el proceso de encuentro entre asistente sexual y personas en situación de discapacidad, con lo cual estos no están de acuerdo. Según su postura, son las personas en situación de discapacidad las que deben reivindicar la temática para la creación de marcos normativos al respecto, más allá de ser los partícipes centrales en definir el rol y figura de la asistencia sexual:

\begin{abstract}
"Son las personas en situación de discapacidad las que deben expresar sus necesidades, decir qué quieren, cómo lo quieren. No son las personas sin discapacidad las que tienen que hacer eso por ellos. Es fundamental que sean los beneficiarios los que tomen las riendas del asunto si quieren que continúe en el largo plazo"16 (Claudine Damarc $^{17}$, presidenta de Cuerpos Solidarios, entrevista realizada en julio de 2017, Lausana, Suiza).
\end{abstract}

En este país, el único cantón donde se ha legalizado la asistencia sexual es Ginebra, no sucediendo lo mismo con el resto de Suiza.

En Francia, las luchas por el reconocimiento en torno a la relación sexualidad-discapacidad comenzaron a hacerse visibles a partir de la Ley de 2005 "Por la igualdad de derechos y oportunidades, participación y ciudadanía de las personas discapacitadas", cuando varias asociaciones de personas en situación de discapacidad empezaron a reclamar la reglamentación de servicios de acompañamiento sexual similares a los de Holanda, Suiza y Dinamarca (CCNE, 2012) En el año 2007 surge el Colectivo discapacidades y sexualidades (CHS), el cual se presenta al Senado en marzo de 2008 solicitando se trate el tema de la asistencia sexual. En abril de 2011 una misión parlamentaria introduce un informe titulado 'Prostitución, la exigencia de responsabilidad: para acabar con el mito del oficio más viejo del mundo', que luego da espacio a que, en junio de 2011, la entonces Ministra de Solidaridad y Cohesión Social, Roselyne Bachelot, en sesión № 118 del 4 de octubre de 2012, plantee: "La sexualidad es una libertad de la cual ningún ser humano adulto debe ser excluido. Somos todos seres sexuados (...). El disfrute sexual que surge del deseo implica lo más íntimo del cuerpo y, por lo tanto, a la persona en todas sus dimensiones sensoriales, emocionales y afectivas" (CCNE, 2012: 3). Y suma a su exposición tres cuestiones: ¿cuáles prestaciones debería ofrecer la sociedad para atenuar las ausencias sentidas por las personas en situación de discapacidad sobre su vida afectiva y sexual y la puesta en marcha de servicios de acompañamiento sexual?, ¿cómo sería la materialización de tales servicios para aquellos/as enmarcados en el derecho a esta compensación desde profesionales del sector sanitario y médico-social?, ¿cuáles serían los medios susceptibles de promover en el personal del sector sanitario y social las buenas prácticas relativas a la vida privada, respeto de la libertad y de la dignidad de las personas en situación de discapacidad? Estas luchas en la arena política fueron acompañadas por personas en situación de discapacidad, familiares y profesionales, que solicitaban un cambio en las prácticas y en el reconocimiento en el derecho y en la solidaridad. Se llega así a un debate que genera contradicciones: ¿ayuda sexual o acompañamiento?, ¿Esta figura sería un "profesional benévolo", un facilitador para permitir la relación sexual de una pareja de personas en situación de discapacidad o para los procesos de autosatisfacción?, ¿los/as ayudantes sexuales estarían asimilados/as a la prostitución, a la asistencia personal o sería

16. Las traducciones de las entrevistas son propias, tanto las realizadas en inglés como en francés. En el presente artículo se pone directamente la versión traducida.

17. Tal como se ha mencionado en el Mapeamiento del campo, tanto Claudine Lamarc, como Joke e Isabelle, más allá de militantes en la temática, son acompañantes sexuales de personas en situación de discapacidad con dependencia severa. Ello implica que las voces de quienes hoy día ejercen este rol aparecen desplegadas en el cuerpo del texto. 
una figura diferenciada de las otras dos? Para superar estas situaciones plantean que debería generarse la figura de la asistencia a la vida sexual como una intervención profesional remunerada con formación específica en la relación sexualidad-discapacidad, pagada por el Estado según el reclamo de varias asociaciones de personas en situación de discapacidad. El Estado quedó así confrontado ante cuestiones de ética deontológica de los derechos y del respeto a los marcos normativos en discapacidad, ante lo cual respondió que no se podía admitir que la ayuda sexual sea un derecho creado que implique una obligación por parte de la sociedad, debiendo quedarse en el marco de las iniciativas individuales (CCNE, 2012).

Según Marcel Nuss, uno de los mayores activistas franceses en torno a esta temática:

"Esta hubiese sido una solución. Llevarlo al plano contractual: yo te doy algo y tú me das algo. El acompañamiento sexual como medio para el ejercicio del derecho a la sexualidad de personas con dependencia severa. $\mathrm{Y}$ digo acompañamiento, no asistencia como en Suiza, ya que ser asistido implica una relación de asimetría. (...). Estamos convencidos de que para cambiar la mirada hay que utilizar la palabra justa" (Marcel Nuss, activista francés, entrevista realizada en julio de 2018, Montpellier, Francia).

En el caso de España, desde comienzos del milenio empiezan las luchas por el reconocimiento para la "diversidad funcional"18 del Foro de Vida Independiente y Divertad. Tal como plantea Antonio Centeno, en la entrevista que se le realizara para la presente investigación en Barcelona, en junio de 2018: "Las conquistas del Foro de Vida Independiente han sido claras. Mucho ha cambiado la mirada y las acciones en torno a la diversidad funcional en España en los últimos años". Algo similar alude Rafael Reoyo, también en entrevista realizada en Barcelona, en junio de 2018, para los fines de la presente investigación: "Claro que ha habido. EI Foro de Vida Independiente, con Romañach a la cabeza, ha generado muchos cambios". El mismo surge en 2001 con el objetivo de llevar a y potenciar en España el movimiento de Vida Independiente surgido en EEUU en 1972. Al concepto de vida independiente, le suman en el título del Foro la palabra divertad, conjunción de diversidad y libertad, ideas medulares para el mismo. Se trata de un espacio cuyo encuentro es a través de internet y se forma por personas en situación de discapacidad para llevar la temática a una "reflexión filosófica y de lucha por los derechos de las personas con diversidad funcional" (Foro de Vida Independiente, 2018: 1) A su vez, forman parte de la Red Europea de Vida Independiente (ENIL). Su filosofía es: ¡Nada sobre nosotros/as sin nosotros/as! (Foro de Vida Independiente, 2018: 2) Es en este marco que comienzan las reivindicaciones en torno a la temática de la sexualidad de las personas en situación de discapacidad.

"La asexuación de las personas con diversidad funcional constituye uno de los pilares del sistema capacitista que las oprime desde tiempos inmemoriales. Eliminando o reduciendo a lo anecdótico la sexualidad, el deseo y el placer, resulta más fácil acotar los horizontes vitales de las personas con diversidad funcional a la mera supervivencia" (Centeno en Foro de Vida Independiente y Divertad, 2017: 1).

El año 2013 parece tener un punto de inflexión en la relación sexualidad-discapacidad en este país, ya que luego de algunas reuniones surgidas entre militantes en situación de discapacidad, profesionales interesados en la temática y familiares, emanan tres miradas que devendrán en la conformación de este rol y figura, fundamentalmente en Cataluña. Por un lado, a través de la persona de Antonio Centeno con su codirección

18. Concepto utilizado por el Foro de Vida Independiente, el cual se ha extendido al campo académico español que refiere a la temática de la discapacidad desde el modelo social. Este concepto de "diversidad funcional" está apareciendo, también, en varios textos latinoamericanos generándose varias controversias. 
en el film 'Yes, we fuck', por otro, la de Rafael Reoyo desde el plano académico y, por el otro, se funda la organización Tandem-Team, de la mano de Francesc Granja. Cada uno de estos actores desde sus distintos espacios y formaciones llevó la temática al tapete, generándose hasta el día de hoy varias formas en las cuales la hacen visible en el país.

En el caso de Inglaterra, tanto en la entrevista realizada a Thomas Shakespeare en Norwich en julio de 2018, como en los diversos datos recabados en torno a la relación sexualidad-discapacidad en dicho país, el primer nombre que surge es el de Tuppy Owens, terapista sexual que, entre otras cosas, crea en 1979 el Outsiders Club, un espacio de encuentro para personas en situación de discapacidad mental y física.

\footnotetext{
"Outsiders es un club social, de apoyo mutuo y citas, dirigido por y para personas con discapacidad social y física. Nuestros miembros tienen una amplia gama de deficiencias, incluidas las discapacidades visuales y auditivas. Nos dicen que solo otras personas con discapacidad realmente los entienden, así que resultan los mejores compañeros. Todos los nuevos miembros son examinados por voluntarios experimentados" (Outsiders Club, 2018: 1).
}

Entrado el nuevo milenio se crea en Inglaterra el TLC Trust Website ampliando los espacios de encuentro para esta población hacia la inclusión de trabajadores/as sexuales. Owens plantea que la creación de este espacio surge a partir de la solicitud de trabajadores/as sexuales, de diversos miembros del Outsiders Club y de la Línea de Ayuda Sexo y Discapacidad. El haber trabajado con esta población desde la creación del Outsiders Club le dio la posibilidad de escuchar sus demandas desde fines de los años 70 , lo que la llevó a crear este espacio y proporcionar una forma segura de encuentros entre personas en situación de discapacidad y trabajadores/as sexuales con experiencia. Plantea que estos espacios brindan a las personas en situación de discapacidad confianza en sí mismas, les permite aprender sus capacidades corporales en torno al placer de sus cuerpos, sentirse amados y satisfechos a través de espacios de intimidad (TLC Trust, 2018).

De una nota periodística de The Guardian del año 2013, se pudo conocer que no sólo por este lado van las luchas por el reconocimiento en Inglaterra. Allí aparecen discursos de personas en situación de discapacidad que reclaman un marco normativo similar al de Holanda: "Ios ciudadanos con discapacidades reciben dinero público para pagar servicios sexuales hasta 12 veces al año" (Ryan, 2013: 1). Sin embargo, se han enfrentado varios activistas en situación de discapacidad a las respuestas de un Estado que les responde que éste "no es un tema prioritario". Desde Scope, una asociación de personas en situación de discapacidad, se plantea:

\footnotetext{
"Tenemos que romper con los tabúes en torno a la discapacidad y la sexualidad. Pero es imposible tener algún tipo de relación si no tienes el soporte que necesitas para salir de la cama, bañarte, vestirte, comer apropiadamente y salir de la casa. Al mismo tiempo, muchas personas en situación de discapacidad están preocupadas en cómo pagar sus cuentas" (Ryan, 2013: 2).
}

En el caso de Bélgica, según lo recabado en la entrevista realizada a Steven De Wirdt en Bruselas, en junio de 2018, se han venido produciendo cambios sustantivos en la sociedad belga en los últimos 25 años en torno a la relación discapacidad-sexualidad.

"Hace 25 años todo lo que concernía a la discapacidad y la sexualidad era un gran tabú. Y eso estaba vinculado a que la mayoría de las instituciones estaban regentadas por la iglesia católica. Hoy día hay fundamentalmente 
educadores, asistentes personales, que plantean que hay mucha frustración en la población con la que trabajan y que necesitan ayuda. (Sin embargo), una cosa es la Bélgica flamenca, que es más abierta, y otra cosa es la Bélgica francesa que continúa teniendo miradas más vinculadas a la sexualidad como lo que se da en el ámbito privado" (Steven De Wirdt, presidente de Aditi, entrevista realizada en junio de 2018, Bruselas, Bélgica).

Aditi existe desde hace una década. Se trata de una organización pequeña de la Bélgica flamenca que ha realizado intensas luchas por el reconocimiento de las personas en situación de discapacidad y su derecho a la sexualidad. Su trabajo se orienta en tres niveles: a nivel de la sociedad, sensibilizando sobre la temática ${ }^{19}$, a nivel del sector profesional de la salud ${ }^{20}$ y a nivel de la asistencia sexual específicamente ${ }^{21}$.

De Wirdt resultó el único entrevistado que directamente planteó la diversidad de propuestas y posturas en torno a la temática en Europa. Ello lo aludió a que cada país debe dar las respuestas singulares a partir de los marcos normativos que cada uno tenga sobre el trabajo sexual.

"En Europa tenemos una red de instituciones que es muy laxa. Se llama EPSEAS y fui yo quien la cree. Hace cuatro o cinco años Aditi comenzó a plantear que era momento de hacer una red europea de las instituciones que trabajaran en la asistencia sexual, de manera de obtener dinero de la Comunidad Europea para la formación de la asistencia sexual. Ese fue el origen de EPSEAS. De ahí nos pusimos en contacto con Tandem-Team en España, con Sheila en Suiza, con Massimo en Italia y otras organizaciones. Pero me parece que no se puede llegar a más que esto porque de verdad cada país tiene una situación muy distinta" (Steven De Wirdt, presidente de Aditi, entrevista realizada en junio de 2018, Bruselas, Bélgica).

En los países donde es legal el trabajo sexual, la potencia para desplegar nuevas proyecciones hacia la asistencia sexual es mucho más amplia que en los países donde no lo es. Ello en el entendido naturalizado de los gobernantes y la sociedad en general de que la asistencia sexual es una figura similar en su rol al trabajo sexual, para lo cual este activista en la temática insiste en generar sensibilización en sus gobernantes y en la sociedad en general.

Holanda, al igual que Dinamarca, son los únicos países que tienen legalizada la figura de la asistencia sexual en todo su territorio nacional, pero porque es legal el trabajo sexual. Sin embargo, aparecen con distancias entre ellos y con el resto de los países europeos en la forma de materializar este rol y figura.

En Holanda, en 1992 comienzan a aparecer las primeras interpelaciones en la esfera de la solidaridad, de la mano de Jeffrey Stalk, en torno a que la sexualidad para las personas en situación de discapacidad era un derecho. Ello surge a partir de una lucha singular en la esfera del derecho de parte de una persona en

19. "La sociedad acá es compleja. Por ejemplo, si preguntamos ahora a personas en la plaza qué piensan en torno a la sexualidad de las personas en situación de discapacidad, más del $80 \%$ va a decir sí, pero eso es un blabla. Si preguntamos en lo concreto, sobre situaciones concretas de personas dependientes responderán que lo tienen que pensar porque nunca habían reflexionado en torno a eso. Por eso hay muchas cosas por hacer todavía en torno a la sensibilización" (Steven De Wirdt, presidente de Aditi, entrevista realizada en junio de 2018, Bruselas, Bélgica).

20. "El $40 \%$ de nuestro tiempo lo dedicamos a la formación de estos trabajadores. Son profesionales de las 180 instituciones que en la Bélgica flamenca trabajan con la discapacidad y la dependencia severa, porque sumamos a las personas mayores en nuestro programa. Pero no sólo les damos formación, sino también respuestas concretas a demandas que nos realicen sobre la sexualidad de las poblaciones con las que trabajan. Por ejemplo, si hay una personas en situación de discapacidad que comienza a masturbarse en la calle, nos llaman a nosotros y nosotros hablamos con sus acompañantes, con los educadores, para darles herramientas para resolver estas situaciones" (Steven De Wirdt, presidente de Aditi, entrevista realizada en junio de 2018, Bruselas, Bélgica).

21. "Damos respuesta a las demandas de las personas en situación de discapacidad, o sus familias, o los profesionales de las instituciones donde están, en lo que concierne a la sexualidad” (Steven De Wirdt, presidente de Aditi, entrevista realizada en junio de 2018, Bruselas, Bélgica). 
situación de discapacidad con dependencia severa en lo mental, que arguye que su sufrimiento se potenciaba al no tener contacto sexual. El Consejo de Estado debió dar cuenta de esta decisión judicial a favor de la personas en situación de discapacidad y se le exigió a la municipalidad de Noordoospolder el pago mensual de lo que en su momento eran 150 florines (hoy día 83 euros) para que una vez al mes, en sesiones de 90 minutos, pudiese tener una 'visita' a lo que se llamó "trabajador de ayuda sexual". A partir de esta situación, comenzó a extenderse la potencialidad de este rol y figura para todo el país, planteando en sus luchas que, en caso de que la persona en situación de discapacidad no pudiese pagar a este trabajador de ayuda sexual, el Estado debía hacerlo. Para ese momento, los argumentos que se ponían sobre la mesa giraban en torno, no sólo al derecho de las personas en situación de discapacidad de tener encuentros sexuales para su bienestar general, sino a que esta población tenía reales dificultades para conocer y entablar relaciones con otras personas. Se funda así, hacia fines de los años 90, la Fundación para Organizar Relaciones Alternativas, comenzando con alrededor de 500 personas en situación de discapacidad que demandaban esta subvención, específicamente otorgada por el sistema de seguridad social del gobierno holandés. Varios reportes de esos años comenzaron a argumentar el beneficio de este sistema, el cual entendían que reducía significativamente la depresión y las tasas de suicidio en la personas en situación de discapacidad. El trabajo de campo realizado en este país quedó remitido a intercambios por mail con algunas trabajadoras sexuales, las cuales tampoco quisieron dar información concreta de este trabajo. Sólo remitieron a documentos donde tomar la información que se expuso.

Algo similar sucedió con Dinamarca desde el discurso de La Central de Discapacidad, organización nacional en torno a las políticas de discapacidad en dicho país. En abril de 2018 se derivó hacia Organizaciones Danesas de Discapacidad, recibiéndose textos solamente en danés para dar cuenta de las luchas por el reconocimiento en torno a esta temática en dicho país y las conquistas logradas.

Más allá no se pudo contar con entrevistas en estos dos países, resultó necesario incorporarlos en esta deconstrucción puesto que desde los otros países donde sí se realizaron entrevistas remitieron a uno y otro insistentemente, ya fuese por concordancia como por discrepancia.

\section{Marcos normativos}

Tal como se ha planteado, no sólo resulta sustantiva la esfera de la solidaridad para el reconocimiento de la relación discapacidad-sexualidad, sino que la esfera del derecho requiere hacerse sustancia para que se generen cambios concretos. Quienes abogan por el reconocimiento normativo del acompañamiento sexual, plantean que las personas en situación de discapacidad deben tener la oportunidad de conocer(se) en su sexualidad en igualdad de condiciones que el resto de la sociedad. Para ello apelan a los apoyos necesarios y las condiciones favorables, entendiendo que el rol y figura del acompañamiento sexual sería sustantivo.

En este sentido, Navarro (2014), plantea tres aspectos fundamentales a ser considerados: el derecho al propio cuerpo, la figura de la asistencia sexual y las diferencias entre asistencia sexual y vida sexual. En relación con el derecho al propio cuerpo, este autor hace referencia a que los cánones estéticos contempo- 
ráneos interpelan sistemáticamente a las personas en situación de discapacidad, por ser considerados sus cuerpos lejos de las normatividades de belleza. En sus planteamientos, este autor arguye que el derecho al propio cuerpo "es la materialización de la personalidad mediante el acceso, exploración, preparación y actividad sexual" (Navarro, 2014: 5). Para ello, debe existir una figura específica que concretice este derecho, siendo la del acompañamiento sexual la que entiende como la adecuada. En cuanto a la asistencia sexual (término que este autor plantea), resulta el espacio entre la asistencia personal y el trabajo sexual, siendo sus funciones las de acompañar a las personas en situación de discapacidad "antes, durante y/o después de las prácticas sexuales con otras personas en todo aquello que no puede hacer sin ayuda" (Navarro, 2014: 6). Muchas veces las personas en situación de discapacidad no pueden experimentar su sexualidad en su pleno uso y disfrute "debido a una diferencia encarnada", por lo que se deben generar los puentes necesarios para que ello ocurra. En relación con las diferencias entre la asistencia sexual y la vida sexual en las personas en situación de discapacidad, este autor se refiere a que históricamente se ha abordado esta temática desde un modelo de discapacidad en el cual la sexualidad se percibía como deseo, pero no como una verdadera necesidad. Para ello, deben darse los apoyos necesarios, de forma tal que la asistencia sexual sea un acuerdo libre entre ambas partes, sin constreñimientos y posibles consecuencias negativas al respecto para quienes lo soliciten y/o lo lleven adelante. Más allá de la legalidad o no, el acompañamiento sexual se realiza, por lo que desde estos países en movimiento por estos derechos apuntan sostenidamente a capacitar a personas que quieran llevar adelante esta actividad.

Por su parte, Nuss (2012) plantea que desde hace algunos años la sexualidad de las personas en situación de discapacidad ha comenzado a hacerse visible, luego de una historia cargada de desexualización y negación de su libido. Ante esta demanda exponencial, urgen respuestas para el reconocimiento de la sexualidad de las personas en situación de discapacidad y la puesta en marcha de dispositivos adecuados para poderla experimentar.

"De hecho, ser uno mismo, estar de acuerdo con uno mismo, conduce a crear su propia norma. Yo tengo mi norma, la que se nutre de mi ética, de mi singularidad y de mis valores personales, en todos los ámbitos: sociales, políticos, afectivos y sexuales. Nadie puede vivir en mi lugar. En este sentido, ser autónomo es ser su propia norma" (Nuss, 2012: 25).

Para este activista francés en situación de discapacidad con dependencia severa, la sexualidad es una libertad incontestable, ya que toda persona es libre de hacer con su cuerpo lo que quiera, más allá se esté en una situación de discapacidad: "Que se tenga o no una discapacidad, nuestra sexualidad y nuestra sensualidad nos pertenece" (Nuss, 2012: 58).

Para conocer los discursos de los marcos normativos de los países europeos en torno a la figura del acompañamiento sexual, no sólo se preguntó en las entrevistas cómo era la situación en su país sino que, además, destacaran lo de algunos otros países. De ahí que, en su concreción, la situación holandesa fue la destacada en el discurso de la mayor parte de los entrevistados.

\footnotetext{
"Según tengo entendido, sólo en Holanda se paga desde el Estado la asistencia sexual. Pero, no es el mismo servicio que damos nosotros. Es un servicio de prostitución. No hay un proyecto, no hay seguimiento, hasta donde sé no hay formación. Para nosotros Holanda no es un modelo a seguir" (Claudine Damarc, presidenta de Cuerpos solidarios, entrevista realizada en julio de 2017, Lausana, Suiza).
} 


\begin{abstract}
"No existe una ley en Holanda que regule la asistencia sexual. Lo que han venido haciendo es que varias comunas, no todas, reembolsan la asistencia sexual. Pero el problema que tienen, me parece, es que no tienen un centro con la experticia como el nuestro para reglar todo lo que hace a la sexualidad y la discapacidad. No tienen formación, cualquiera puede ser asistente sexual. Por eso me parece que se confunde con la prostitución. Por eso es necesario un marco normativo que regule la asistencia sexual" (Steven De Wirdt, presidente de Aditi, entrevista realizada en junio de 2018, Bruselas, Bélgica).
\end{abstract}

Los diversos discursos recogidos en torno a la ley holandesa desde los entrevistados en el trabajo de campo realizado en Europa se basan, por lo general, en relatos encarnados en apreciaciones que muchas veces distan de lo que de hecho sucede en dicho país. En Holanda no hay una ley específica en torno al acompañamiento sexual, sino que el trabajo sexual está legalizado, por lo cual corren por la misma vía. Lo que sí se ha logrado, a partir de las luchas por el reconocimiento, es que el Estado dé como parte de las prestaciones a las personas en situación de discapacidad la posibilidad de hacer uso de acompañantes sexuales.

El cantón de Ginebra, en Suiza, sin embargo, tiene un marco normativo de cierta forma específico sobre este rol y figura, más allá de que pueda entenderse como una asimilación a la de trabajo sexual.

"A nivel de la ley, la asistencia sexual en Suiza está asimilada a la prostitución, la cual es legal. Hay una excepción en el cantón de Ginebra (las leyes son cantonales, no nacionales), donde la asistencia sexual no es considerada dentro de la prostitución, pero tampoco tiene un status real" (Claudine Damarc, presidenta de Cuerpos solidarios, entrevista realizada en julio de 2017, Lausana, Suiza).

"El cantón de Ginebra, por ejemplo, plantea que la asistencia sexual no es prostitución, por lo que quienes ejercen la asistencia sexual no deben inscribirse en la Policía. Pero esa es una excepcionalidad, por lo general se asimila normativamente a la prostitución" (Sheila Waremburg, presidenta de Sexual Understanding, entrevista realizada en julio de 2017, Annecy, Francia).

En la particularidad francesa, a partir de sus postulados de libertad, igualdad y fraternidad, las discusiones en torno a esta temática corren, no sólo por la asimilación del acompañamiento sexual a la prostitución, sino a la generación de marcos normativos discriminatorios, más allá de que estos sean por discriminación positiva.

"Yo me pregunto, ¿es necesario tener una ley específica para las personas en situación de discapacidad para que lleguen a una asistencia sexual? ¿No habría ahí una desigualdad entre las personas en situación de discapacidad y los otros? En la ley francesa no queremos hacer discriminación, y eso me parece bien. Yo prefiero que la ley sea la misma para todo el mundo. Quizá, una excepción que consideraría sería la de esa cuestión de los intermediarios y su punición, porque lo que queremos es asegurarnos de que la persona en situación de discapacidad esté segura (...). Ese sería el único detalle que quisiera que la ley tuviera" (Sheila Waremburg, presidenta de Sexual Understanding, entrevista realizada en julio de 2017, Annecy, Francia).

Tal como plantea Corbet (2014), el acompañamiento sexual continúa siendo una profesión muy marginalizada y tabú en Francia, asociándola aún a la prostitución. En este país, la prostitución sanciona a quien solicita el servicio, no a quien ejerce el trabajo sexual.

"En Francia, la prostitución no es ilegal, salvo para los clientes, quienes pueden correr el riesgo de ser penados (...). La persona en situación de discapacidad debe saber que ese riesgo existe. La otra dificultad es que cualquier 
persona que sea intermediario, como yo por ejemplo que soy sexóloga, puede ser considerada como un proxeneta y, por lo tanto, recibir castigo. Mismo si no gana dinero. Los padres, por ejemplo, que nos contactan, pueden ser considerados, según la ley, como proxenetas. Esa es una gran dificultad" (Sheila Waremburg, presidenta de Sexual Understanding, entrevista realizada en julio de 2017, Annecy, Francia).

En relación con la ley belga, en agosto de 2018 entró al Parlamento una ley específica sobre el acompañamiento sexual. De esta manera, la Bélgica flamenca sería, al igual que el cantón de Ginebra en Suiza, los únicos países europeos que en alguno de sus territorios tendrían legalizado este rol y figura.

"Uno de los partidos políticos de la Bélgica flamenca escribió un protocolo y ya hay otros tres partidos políticos que lo suscriben. Falta uno, que es la mayoría. En caso de aprobarse, el protocolo que creamos y llevamos adelante será oficial para toda la parte flamenca. Como nuestro país es federado y se divide entre la parte flamenca y la francesa, primero se aprobaría en la parte flamenca y luego pasaría a ser ley nacional. Pero ese es un segundo paso (...). Por ahora, no hay ningún país en Europa que tenga un marco jurídico en torno a la asistencia sexual. Si la ley que estamos presentando el mes próximo se aprueba, seríamos los primeros" (Steven De Wirdt, presidente de Aditi, entrevista realizada en junio de 2018, Bruselas, Bélgica).

El proyecto de ley presentado para su debate a nivel parlamentario fue realizado por Aditi y tres universidades belgas, creando un marco jurídico en el cual el acompañamiento sexual estuviese contextualizado en la atención en salud. Fue el trabajo de una década en la arena política para dar razones sustantivas de que se trata de una ley favorable, tanto para las personas en situación de discapacidad como para quienes ejercen el acompañamiento sexual. En su especificidad, este marco jurídico se refiere a la asistencia sexual, no al asistente sexual; es decir, se refiere a la figura genérica, no al rol concreto.

"Si creábamos un marco jurídico para la persona asistente sexual se debería cambiar la ley en torno a la prostitución, lo que llevaría de nuevo a la confusión de la asistencia sexual y la prostitución como si fueran lo mismo. Por eso creamos un marco jurídico para la acción, para la asistencia sexual. Este marco explica que, en principio, se trata de una cuestión de derechos y de política, ya que sin este hay una enorme población frustrada y, a su vez, de todas maneras, se hace sin los cuidados y formación necesarios" (Steven De Wirdt, presidente de Aditi, entrevista realizada en junio de 2018, Bruselas, Bélgica).

La situación española de 'alegalidad' al trabajo sexual genera espacios para poder desplegar el rol y figura del acompañamiento sexual. En relación con ello, de los entrevistados de dicho país aparecen discursos contrapuestos.

"Los marcos normativos pueden constreñir y utilizarse mal, más que nada porque protocoliza la sexualidad. Tiene que haber libertad para ejercer la sexualidad" (Rafael Reoyo, académico y activista, entrevista realizada en junio de 2018, Barcelona, España).

"En España no es legal, eso ayudaría mucho ya que enmarca y genera derechos y obligaciones. Uno tiene a quien recurrir en caso de que no se cumpla con lo acordado" (Antonio Centeno, activista en la temática, entrevista realizada en junio de 2018, Barcelona, España).

En el contexto de esta alegalidad, Tandem-Team encuentra el espacio para trabajar como puente entre el/ la acompañante íntimo y erótico (según su forma de nombrar) y la/s personas en situación de discapacidad. 
Más allá de este nexo inicial, los/as acompañantes íntimos y eróticos firman un documento de exoneración de responsabilidades y de confidencialidad con esta organización, ya que si no se podría relacionar con el proxenetismo. Según los discursos recabados en la entrevista con Francesc Granja, presidente de TandemTeam, realizada en junio de 2018 en Barcelona, más allá no se responsabilicen legalmente de lo que suceda en los acuerdos generados entre personas en situación de discapacidad y el/la asistente íntimo y erótico, mantienen conversaciones con estos durante todo el proceso inicial del protocolo que implementan para que estos encuentros se materialicen. Esta situación de alegalidad aparece como única en los discursos recabados en España, no habiendo otro país europeo que se autorreconozca en esa posición.

En relación con Inglaterra, el pago a trabajadores/as sexuales no está penado por la ley, pero sí es ilegal solicitar servicios sexuales. Para analizar la temática en este país, Thomas Shakespeare, en la entrevista que se le realizara en Norwich en julio de 2018, comenzó su recorrido normativo por la asistencia personal.

\footnotetext{
"La asistencia personal empezó en Inglaterra en los años 70 cuando comenzó el proceso de desinstitucionalización. En 1996 se cambió el marco legal (...), el Estado comenzó a tener obligaciones para proveer a las personas de asistentes personales. No asistentes sexuales, sino asistentes personales. Entonces todo el mundo empezó a presentarse como asistente personal (...). Hoy día la asistencia personal no está regulada, por lo que es invisible. ¿Se sabe lo que pasa dentro de cada casa? No. Porque lo que está regulado tiene inspecciones y eso da garantías, pero lo que pasa dentro de los hogares no" (Thomas Shakespeare, activista y académico, entrevista realizada en julio de 2018, Norwich, Inglaterra).
}

Con estos cambios en las prestaciones para las personas en situación de discapacidad en Inglaterra, por un lado, quienes ejercen la asistencia personal no son controlados en sus espacios laborales, lo cual quita garantías tanto a estos/as trabajadores/as como a las personas en situación de discapacidad. Por otro lado, queda el margen de que la asistencia personal pueda ser confundida con la asistencia sexual, lo cual puede ser positivo o negativo, según la situación concreta.

Por otra parte, al ser ilegal la prostitución en Inglaterra, aparecen dudas similares a las planteadas en Francia sobre la discriminación positiva de hacer una ley específica al respecto para esta población.

"La prostitución es ilegal en Inglaterra. ¿Debería legalizarse para que las personas discapacitadas tuviesen esta figura de la asistencia sexual? Sería mejor legalizar la prostitución y se soluciona el problema” (Thomas Shakespeare, activista y académico, entrevista realizada en julio de 2018, Norwich, Inglaterra).

A partir de estos datos recabados en las entrevistas realizadas en Europa, y más allá de las idealizaciones en torno a ciertos países y sus marcos normativos, en concreto, sólo es legal como figura específica de asistencia sexual en el cantón suizo de Ginebra. La Bélgica flamenca se encuentra en estos momentos analizando en su Parlamento el proyecto de ley realizado y elevado por Aditi en conjunto con tres universidades para darle legalidad a esta figura. En caso de lograrlo, habría que dar un segundo paso hacia la extensión a todo su territorio nacional, encontrando importantes resistencias en la Bélgica francesa, por su "carga católica".

Como cierre, se presenta un cuadro comparativo de la información recabada en los países europeos donde se realizaron entrevistas. 


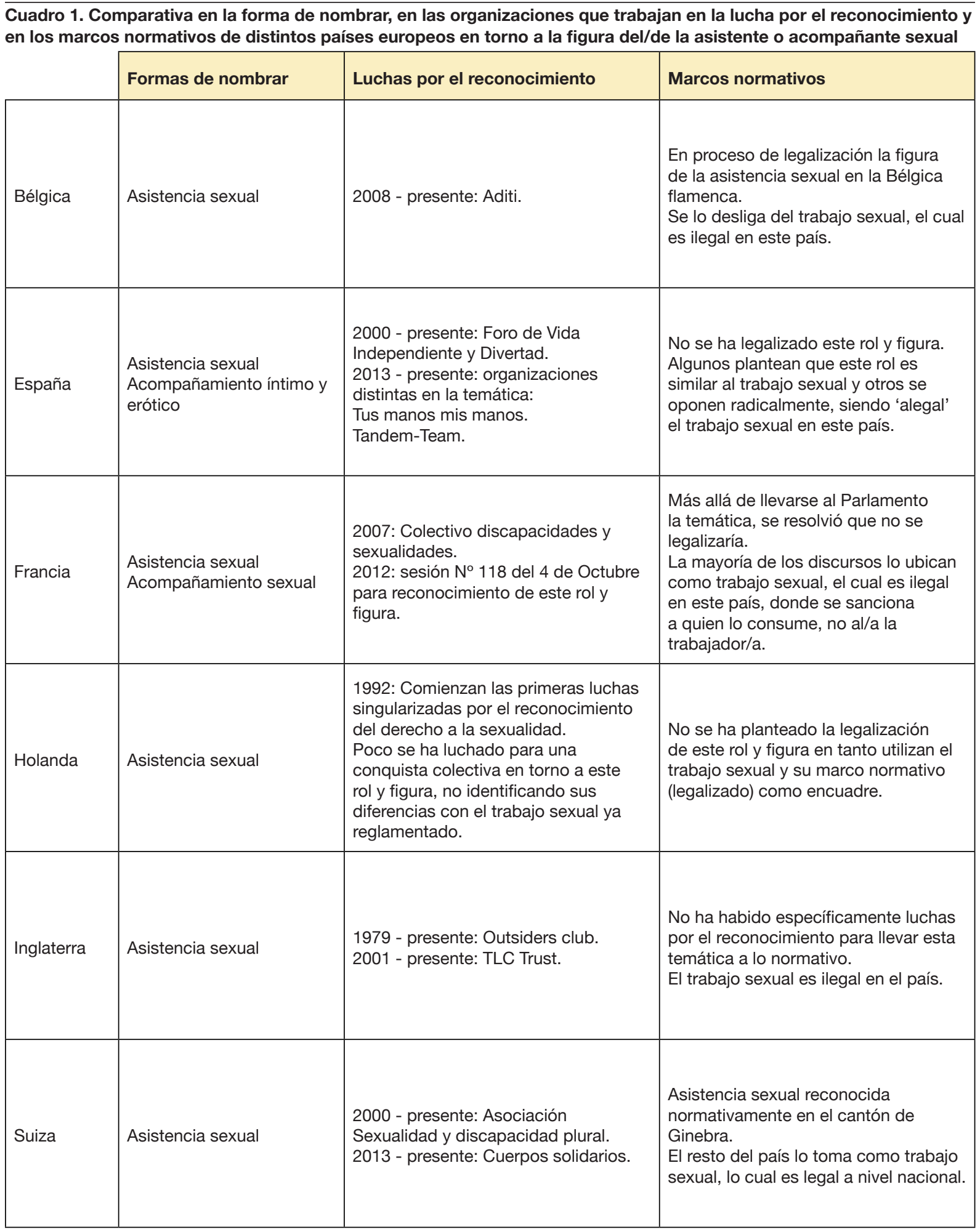

Fuente: elaboración propia a partir del trabajo de campo llevado adelante en Europa. 


\section{Reflexiones finales}

A través de las líneas recorridas en el presente artículo, se llevó adelante uno de los rodeos analíticos devenidos de la investigación 'Cuerpos vivos, cuerpos sentidos. Acompañamiento sexual para personas en situación de discapacidad con dependencia severa', el cual versó en las luchas por el reconocimiento y los marcos normativos en torno a la figura y rol del acompañamiento sexual para personas en situación de discapacidad. La metodología que se utilizó en esta parte de la investigación fue la de entrevistas en profundidad a diversos referentes en la temática en los países europeos que se nuclean a través de EPSEAS, a saber: Suiza, Bélgica, Holanda, Francia, España e Inglaterra.

Se optó por especificar este rodeo analítico para comenzar a desandar la relación entre Estado y sociedad civil organizada, poniendo el foco en las luces y sombras que han aparecido en la última década a partir de las luchas por el reconocimiento de asociaciones civiles de personas en situación de discapacidad en torno a la relación sexualidad-discapacidad, con especificidad en el acompañamiento sexual para esta población, y las respuestas dadas por los Estados de los diversos países analizados.

Algunos de los aspectos sustantivos que se entiende necesario dejar en claro en este artículo se orientan a: encuentros y desencuentros en torno a la temática en los distintos países, luchas por el reconocimiento singulares y/o colectivas, y posicionamientos de los Estados ante estas luchas.

En lo que respecta a los encuentros y desencuentros en torno a la temática, cada país pareciera poner el foco en distintos aspectos de este rol y figura. Esto es, la distinción entre acompañamiento sexual y prostitución; situaciones de legalidad o ilegalidad en el ejercicio del trabajo sexual y las represalias estatales al respecto; quién debería pagar esta figura (Estado o particulares), etc. Para estos y otros aspectos que han ido apareciendo a lo largo de las entrevistas que se realizaron en Europa entre junio de 2017 y julio de 2018 , queda la sensación de que son variadas las aristas hacia las cuales la temática puede desencadenar y que no es tan sencillo llegar a un consenso entre lo que se quiere, cómo se quiere y de qué manera.

En lo que hace a las luchas por el reconocimiento singulares y/o colectivas, cabe destacar el poco o nulo contacto existente entre los referentes de los distintos países. Más allá de conocimientos personales entre el referente belga y uno de los catalanes, y la referente suiza con la referente francesa, los demás no se conocen entre ellos y, por ende, no conocen las propuestas en danza en la última década. Más allá de los esfuerzos llevados adelante por De Weirdt de llegar a hacer un espacio de encuentro virtual como lo es EPSEAS, pareciera que las luchas colectivas por el reconocimiento quedan relegadas a las fuerzas singularizadas de quienes proponen una y otra cosa. Se entiende que ello sea parte de la explicación de por qué a una década de haber comenzado este movimiento en varios países europeos, sólo el cantón de Ginebra tiene aprobado legalmente la figura del acompañamiento sexual, la Bélgica flamenca haya ingresado el proyecto de ley al parlamento, y que en Holanda y Dinamarca de hecho se dé por asimilarlo a la prostitución que es legal en dichos países. En este sentido, la ausencia de luchas colectivas de los distintos movimientos europeos en torno a la temática posiblemente pueda estar incidiendo en la casi ausencia de reconocimiento de este derecho que se pretende quede materializado normativamente.

El posicionamiento de los estados en relación con la temática se entiende termina yendo en consonancia al impacto que estas luchas por el reconocimiento están teniendo, más que nada disgregadas en distintos 
espacios singularizados. Posiblemente, si se pudiese recuperar la idea de De Weirdt a través de EPSEAS y su reconocimiento y financiación por parte de la Comunidad Europea, los esfuerzos singularizados se verían potenciados por los esfuerzos colectivos. Se entiende que la Comunidad Europea tiene este enorme valor que debería interiorizarse para exteriorizar luchas colectivas más allá de las singularidades de grupos o países que llevan adelante estos esfuerzos.

En síntesis, se trata de una temática que se encuentra en proceso de reflexión ${ }^{22}$ y visibilización, con diversas propuestas en torno a lo que sería el rol y figura del acompañamiento sexual para personas en situación de discapacidad con dependencia severa. La potencia europea de poder nuclear los diversos esfuerzos singulares hacia la lucha por el reconocimiento colectivo de este rol y figura se entiende que es una puerta que está aún entornada y que sería sustancial abrir. Lo colectivo siempre tiene más fuerza que lo singular, en el tema que se trate, por lo que apelar a ello se entiende sustancial en este entramado.

22. En otros artículos en proceso de elaboración se están realizando rodeos analítico-reflexivos en torno a: el rol del/de la acompañante sexual, las distancias con el trabajo sexual, los acuerdos de trabajo y pagos, las formas de nombrar. Ello mediado por análisis desde la teoría crip, la cual incluye aspectos de la teoría queer, lo que habilita al estudio de la temática desde perspectivas feministas y la incorporación de la mirada desde LGTBIQ. 


\section{Referencias bibliográficas}

CCNE (Comité Consultatif National d'Éthique) (2012): Vie affective et sexuelle des personnes handicapées Question de l'assistance sexuelle (en línea). <https://www.ccne-ethique.fr/sites/default/files/publications/avis_ ndeg118.pdf >, acceso 3 de febrero de 2018.

Corbet, E. (1994): "Violences en institution. A la recherche d'outils de prévention". Handicaps et Inadaptations, 6 : 67-77.

Corps Solidaires (2017): Corps Solidaires (en línea). <http://corps-solidaires.ch>, acceso 5 de junio de 2017.

Foro de Vida Independiente y Divertad (2018): ¿Qué es el FVID? (en línea). <http://forovidaindependiente.org/ que-es-el-fvid/>, acceso 24 de junio de 2018.

Foro de Vida Independiente y Divertad (2017): Mis manos, tus manos (en línea). <http://forovidaindependiente. org/mis-manos-tus-manos/>, acceso 24 de junio de 2018.

Foucault, M. (1998): Los anormales. México: FCE.

Fraser, N. (2000): “Nuevas reflexiones sobre el reconocimiento". New Left Review, 4: 55-68.

Honneth, A. (1997): La lucha por el reconocimiento. Por una gramática moral de los conflictos sociales. Barcelona: Grijalbo.

MIDES (2014): Cuidados como Sistema, Propuesta para un modelo solidario y corresponsable de cuidados en Uruguay. Montevideo: Ministerio de Desarrollo Social.

Míguez, M. N. et al. (2015): Infancia y Discapacidad. Una mirada desde las ciencias sociales en clave de derechos. Montevideo: Unicef.

Naciones Unidas (2006): Convención Internacional sobre los Derechos de las Personas con Discapacidad. Nueva York: ONU.

Navarro, S. (2014): "El asistente sexual para personas con discapacidad, ¿una figura alegal?". En 1er Congreso Internacional Virtual sobre discapacidad y Derechos Humanos. Barcelona: Red Iberoamericana de Expertos sobre la Convención de los Derechos de las Personas con Discapacidad.

Nuss, M. (2012): Je veux faire l'amour. Handicap, sexualité, liberté. París: Autrement.

Outsiders Club (2018): Outsiders (en línea). <http://www.outsiders.org.uk/outsidersclub/>, acceso 24 de junio de 2018.

Ryan, F. (2013): I want a world where disabled people are valid sexual partners (en línea). <https://www.theguardian.com/society/2013/feb/12/disabled-people-valid-sexual-partners>, acceso 11 de noviembre de 2018.

Sartre, J. P. (2000): Crítica de la razón dialéctica. Buenos Aires: Losada.

Sartre, J. P. (1947): El existencialismo es un humanismo. Buenos Aires: Sur.

Shakespeare, T. (2018): Disability. The basics. Londres: Routeledge.

Tandem-Team (2016): Sexualidad en diversidad funcional o intelectual. Barcelona: Tandem-Team.

TLC Trust (2018): Welcome (en línea). <http://tlc-trust.org.uk>, acceso 24 de junio de 2018.

Universo Devotee (2016): Universo Devotee (en línea). <http://universodevotee.blogspot.com>, acceso 29 de junio de 2018. 